\title{
Evaluation of the Success Rate of Ultrasound-guided Transjugular Liver Biopsy (TJLB) and the Associated Complications
}

\author{
Masoud Pezeshki Rad ${ }^{1}$, Hamidreza Sima², Reza Khazaeeian³ ${ }^{3}$ Mahyar Mohammadifard ${ }^{4}$
}

${ }^{1}$ Associate Professor of Radiology, Department of Radiology, Imam Reza Hospital, School of Medicine, Mashhad University of Medical Sciences, Mashhad, Iran

2 Assistant Professor of Gastroenterology, Department of Internal Medicine, Imam Reza Hospital, School of Medicine, Mashhad University of Medical Sciences, Mashhad, Iran

${ }^{3}$ Radiologist, Department of Radiology, Imam Reza Hospital, Mashhad University of Medical Sciences, Mashhad, Iran

${ }^{4}$ Associate Professor of Radiology, Department of Radiology, Imam Reza Hospital, Birjand University of Medical Sciences, Birjand, Iran

\section{Type of article: Original}

\begin{abstract}
Introduction: Transjugular liver biopsy (TJLB) is a diagnostic radiological intervention, applied to patients with contraindications to percutaneous or laparoscopic liver biopsy. The aim of this study was to determine the safety, effectiveness, complications, and pathological impact of TJLB.

Methods: This cross-sectional study was conducted on 24 consecutive patients, undergoing TJLB at Imam Reza Hospital, affiliated to Mashhad University of Medical Sciences, from April 2010 to March 2011. The inclusion criteria were coagulopathy, thrombocytopenia, and ascites. TJLB was performed by a radiologist, and a guide wire was inserted in the right jugular vein. Right atrium and middle hepatic vein were catheterized, and then, Cook Quick-Core Biopsy Needle was used by ultrasound guidance. Data analysis was performed by SPSS version 16.

Results: The sample size included 24 subjects ( $54 \%$ males and $46 \%$ females). Success rate of TJLB was reported as $87.5 \%$ and the intervention duration was $23.89 \pm 9.34 \mathrm{~min}$. No complications were reported in $37.5 \%$ of the patients; however, pain in the right upper quadrant (33.3\%), leakage of contrast substance into the liver capsule $(4.2 \%)$, and diffuse abdominal pain $(4.2 \%)$. In $83 \%$ of biopsies, suitable samples were obtained for histopathological diagnosis, and $13 \%$ of patients were excluded from the study. According to the results, the mean central port track (CPT) was $6.75 \pm 2.95$.

Conclusion: TJLB is a relatively effective and safe procedure for patients with contraindications to percutaneous liver biopsy.

Keywords: Transjugular liver biopsy, Histopathological sample, Ultrasound guidance
\end{abstract}

\section{Introduction}

Given the segmental anatomy of liver, use of ultrasound in this region has 3 limitations: 1) Several segments are adjacent to the same hepatic vein; 2) Right hepatic vein and its branches are not always visible in the caudal region of the right lobe; and 3) Hepatic veins have abundant anatomic variations (1). Some structures such as Glisson's capsule cannot be seen via ultrasound. However, when liver is surrounded by ascites and ultrasound beam is perpendicular to the surface of the liver, a linear echo can be seen throughout the liver. Moreover, in the absence of ascites on scan, a similar linear echo is seen along the liver, using a 5 to $7.5 \mathrm{MHz}$ linear probe. Also, the falciform ligament cannot be seen on the CT scan unless it is surrounded by ascites or lipid tissues or fibers are not present in

\section{Corresponding author:}

Associate Professor Dr. Mahyar Mohammadifard, Department of Radiology, Imam Reza Hospital, Birjand University of Medical Sciences, Birjand, Iran.

Tel/Fax: 00985138414499, Email: Mahyarmohammadifard@yahoo.com

Received: June 14, 2016, Accepted: October 27, 2016, Published: December 2016

iThenticate screening: August 14, 2016, English editing: November 16, 2016, Quality control: December 02, 2016

(C) 2016 The Authors. This is an open access article under the terms of the Creative Commons Attribution-NonCommercialNoDerivs License, which permits use and distribution in any medium, provided the original work is properly cited, the use is non-commercial and no modifications or adaptations are made. 
the ligament; in these cases, this ligament is visible as a linear structure or a linear hyperechoic band within the left intersegmental fissure; some other ligaments of the liver can be only seen when the liver is surrounded by ascites (hepatoduodenal, hepatogastric, coronary, and right/and left triangular ligaments) (2). Accurate measurement of liver size, due to the limited available field of vision in ultrasound, is difficult, especially when the liver is enlarged. The most widely used method of liver length measurement is the sagittal approach in the mid-clavicular line, which is usually in correspondence with the mid-hepatic line. If the length of the liver in the midclavicular line is more than $15 \mathrm{~cm}$, hepatomegaly should be considered (3). Generally, liver imaging is performed in order to meet four objectives: 1) evaluation of the causes of hepatomegaly or liver-localized tumors; 2) detecting tumors (primary or metastatic tumors); 3) confirming or rejecting inflammatory or parasitic diseases of the liver; and 4) planning for medical, radiological, or surgical treatments (4). Liver biopsy is the most specific test to assess the nature and severity of liver disease (5), and can be helpful in treatment management and planning. There are several ways to obtain a liver biopsy, among which biopsy through the skin is the most common. However, some contraindications such as coagulation disorders have been reported in this method. In these cases, transjugular liver biopsy (TJLB) can be performed (6). The advantage of this method is that it is not necessary to pierce into the liver capsule. Moreover, in case of bleeding during biopsy, blood enters the patient's venous system and his/her hemodynamics will not be affected. In addition, in this procedure, after finding suitable pathways, we can use other diagnostic procedures such as the measurement of hepatic venous pressure gradient (7); we can also follow other remedial measures such as placing a shunt between the portal and hepatic veins (or other necessary measures) (8). The specific objective of this study was to determine the safety, effectiveness, complications, and pathological impact of TJLB.

\section{Material and Methods}

\subsection{Setting and participants}

This cross-sectional study population consisted of patients with indications for TJLB, who were referred to the radiology department of Imam Reza Hospital, Mashhad, Iran, 2014-2015. Considering the patients' contraindications to routine skin biopsy and the necessity of liver biopsy for disease management and treatment, TJLB was performed. The study conditions were described for the patients, and informed consents were obtained.

\subsection{Exclusion criteria}

The exclusion criteria were as follows: 1) lack of platelet dysfunction; 2) prothrombin duration more than 22 seconds; 3) scar on the neck, especially on the jugular vein; 4) lack of contraindications to percutaneous biopsy; and 5) unfeasibility of performing TJLB.

\subsection{Instrument and data collection steps}

In this study, data were collected via questionnaires and observation. Siemens-Elema radiological device (1980, Hamburg, Germany) was utilized, which was equipped with two separate tubes with simultaneous exposure of faciolateral regions. Therefore, fluoroscopic and angiographic imaging were simultaneously performed without transferring the patient from one bed to another. Also, a Doppler ultrasound machine (SonoSite M-Turbo, SonoSite, Bothell, WA) was used in this study. Before the biopsy, patients with indications for TJLB were admitted to the gastrointestinal division of internal medicine department of Imam Reza Hospital. After preliminary analysis by a subspecialist of gastroenterology, the patients were referred to the radiology department. After six hours of fasting, patients were transferred to the angiography room at the radiology department, and were continuously monitored by nurses in terms of vital signs and heart function. The participants laid down on the fluoroscopy bed in a head-down position. Ultrasound-guided intervention was performed by an experienced radiologist. A pillow was placed under the patient's neck to allow sufficient neck extension; therefore, the radiologist had access to the jugular vein. Afterwards, the location of the internal jugular vein on the right side of the neck was identified. By using $2 \mathrm{cc}$ syringes, $1 \mathrm{cc}$ of lidocaine $2 \%$ was cautiously injected for local anesthesia around the jugular vein (injection should not enter the blood, since it may lead to arrhythmia) (Figure 1). This procedure was performed by a radiologist with ultrasound guidance. A sheath was properly placed in the jugular vein. A guide wire was inserted, which crossed the path of superior vena cava, right atrium, and inferior vena cava. All the mentioned procedures were controlled via fluoroscopy by the radiologist to prevent the subintimal placement of the catheter, given the associated risks for the patient. After putting the catheter in the correct position, a specific biopsy needle was inserted by a radiologist. By maintaining an appropriate distance from the liver capsules, the required ultrasound-guided biopsy specimens were obtained. During this procedure, radiologist distanced himself from the needle and liver capsule, since in cases of capsule rupture, a major complication might occur and the patient might experience severe peritoneal bleeding; presumably, the patient might die since the bleeding is usually uncontrollable (Figure 2). Tissue samples were obtained from patients and reviewed by radiologists (macroscopic review). The sample size and quantity, as well as 
the initial quality (in terms of damage), were checked, and if the sample size was not adequate or the sample was fragmented, another biopsy was performed around the site of the previous biopsy, following the same procedure. In this study, after each sampling, the sample was maintained in saline and immediately given to a pathologist. Then, the harvested area was controlled via fluoroscopy and contrast injection and possible complications were reviewed. After sampling, the perforated skin regions were compressed for one minute to prevent bleeding and hematoma in the neck, which might put pressure on the airway. Abdominal ultrasound was performed to check the presence of free fluid or hematoma in the liver and peritoneal cavity. The obtained results were recorded, and nurses monitored and recorded the vital signs for 24 hours. Moreover, other symptoms such as abdominal pain, nausea, vomiting, and shortness of breath, chest pain, and neck pain were evaluated and recorded. Twenty-four hours after the biopsy, patients were visited by a gastroenterologist and abdominal sonography was performed again for evaluating the probability of any complications. Finally, the histological report was prepared by the pathologist, which included the sample size, rate of sample injury, and the count of complete portal tract (CPT); then, the final diagnosis was made.

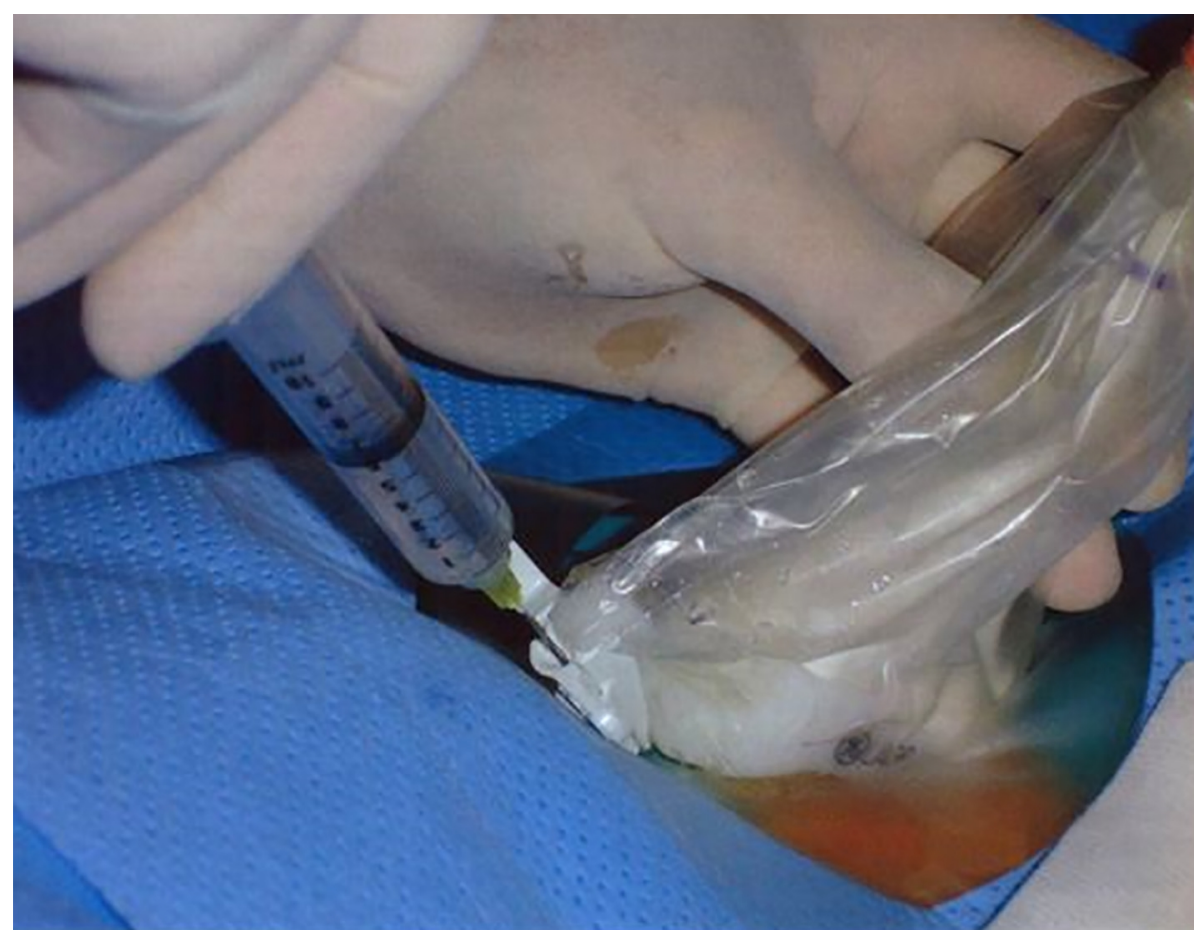

Figure 1. Administration of local anesthesia for ultrasound-guided biopsy of the right internal jugular vein

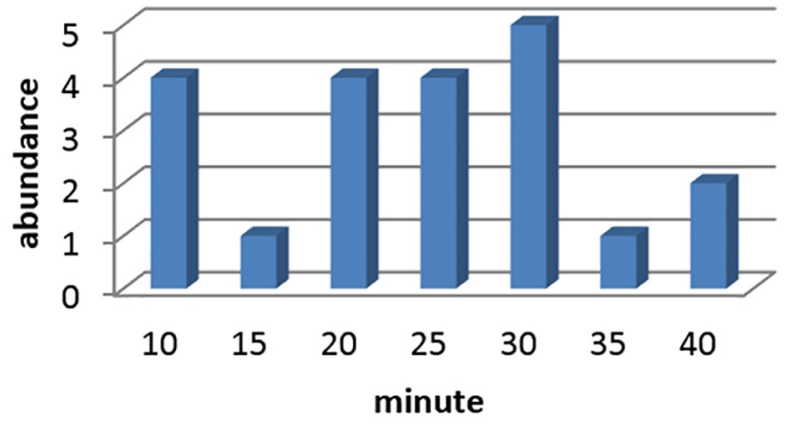

Figure 2. The distribution of intervention duration among patients

\subsection{Statistical analysis}

The collected data from the questionnaires and pathological reports were entered into SPSS version 16 (SPSS Inc., Chicago, Illinois, USA). Chi-square test was applied for measuring the statistical difference between the groups. In all the tests, $\mathrm{p}$-value less than 0.05 were considered statistically significant. 


\section{Results}

In this study, 24 patients ( 13 males and 11 females) were included. The mean age of the patients was 25.35 years, with a standard deviation of 9.78. The subjects were within the age range of 19-57 (70.8\%) years and $7(29.2 \%)$ patients were younger than 29 years. In the present study, $95.8 \%$ of the patients underwent this diagnostic method due to platelet coagulation disorders and platelet count $<30,000$. Only in 1 patient $(4.2 \%)$, this procedure was performed due to hemophilia. In our study, the biopsy was successful in 21 patients $(87 \%)$. However, due to sustained cardiac arrhythmia (in two patients) and unfeasibility of catheterization of the right hepatic vein (in one patient), the procedure was unsuccessful in 3 patients (13\%). Figure 2 shows the duration of intervention (in minutes). According to this diagram, in 5 patients, the intervention duration lasted 30 minutes. The mean and standard deviation of the intervention duration were 23.80 and 9.34, respectively. Table 1 indicates the frequency distribution of complications in the studied patients. In this study, there were no major complications in $91.7 \%$ of the patients; however, minor complications were reported in $62.5 \%$ of the subjects. Three patients $(12.5 \%)$ had neck pain at the time of puncture, eight patients $(33.3 \%)$ had transient pain in the liver area while doing the biopsy and one subject (2.4\%) had abdominal pain after the biopsy; also in one patient, there was contrast media extravasations under the liver capsule.

Table 1. The frequency distribution of complications in patients after the intervention

\begin{tabular}{|l|l|l|l|}
\hline Complication & $\mathrm{n}$ & $\%$ \\
\hline \multirow{4}{*}{ Minor } & Without complications & 9 & 5.37 \\
\cline { 2 - 4 } & Neck pain at the time of puncture & 3 & 5.12 \\
\cline { 2 - 4 } & Contrast media extravasation under liver capsule & 1 & 2.4 \\
\cline { 2 - 4 } & Transient pain in the liver during the biopsy & 8 & 3.33 \\
\cline { 2 - 4 } & Abdominal pain after the biopsy & 1 & 2.4 \\
\hline Major & Sustained cardiac arrhythmia & 2 & 3.8 \\
\hline
\end{tabular}

Two patients $(8.3 \%)$ had sustained cardiac arrhythmia, which was the only major complication among patients. After the intervention, abdominal ultrasound was performed for all patients, who showed no clinical indications. Also, all the patients were under consistent care for 24 hours after the intervention in order to prevent any complications. In most patients with successful sampling, two biopsy specimens were taken (11 patients); also, in 8 and 2 patients, 1 and 3 biopsies were performed, respectively. The biopsies were suitable for pathological reports in 20 patients; in one patient, the biopsy was not suitable for the pathological report. Three patients were excluded from the study due to sustained cardiac arrhythmia (in two patients) and unfeasibility of catheterization of the right hepatic vein (in one patient). Figure 3 shows the frequency of CPT count in the biopsies. According to this diagram, the maximum CPT count in the biopsies was 6 (with a frequency of 4), followed by 5 and 7 (each with a frequency of 3). The mean \pm standard deviation of CPT count was $6.75 \pm 2.95$. According to liver biopsies, mild inflammation ( 8 patients) and cirrhosis of the liver ( 8 patients, maximum frequency) were diagnosed; autoimmune hepatitis was the next most prevalent diagnosis, reported in 4 cases.

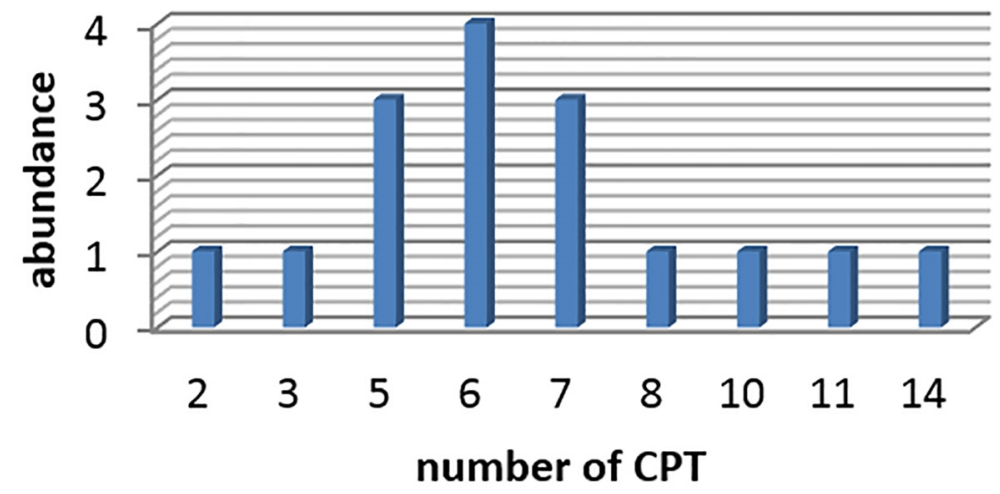

Figure 3. Frequency of CPT count in the biopsies

It should be noted that in pathological reports, more than one diagnosis was mentioned for some patients. Neck pain during puncture was observed in only 3 women (no males). Transient pain in the liver area during biopsy was seen in seven men and one woman. However, the relationship between the two variables was not statistically significant 
$(\mathrm{p}=0.090)$. The duration of intervention ranged between 10-40 minutes while most of the procedures lasted for 40 minutes (88\%), but despite the differences between the two genders in duration, no significant statistical relationship was observed between these variables $(\mathrm{p}=0.126)$. The most common complication was transient pain in the liver during biopsy, which had the highest incidence in the age groups of 30-33 years and $>44$ years. However, no significant statistical association was observed between these two variables $(\mathrm{p}=0.243)$.

\section{Discussion}

In this study, sampling was successful in most patients (87\%) without causing any major complications; only 3 patients (13\%) experienced biopsy-related complications. The intervention duration was relatively short and in most patients, biopsies were helpful for diagnosis with proper CPT count. Given that simple random sampling was employed in this study, $54 \%$ of the subjects were males and $46 \%$ were females; gender distribution was similar to that of Cholongitas' study (55\% male and 44\% female), which assessed the performance of TJLB in patients without liver transplantation (7). Contrarily, in Nassiri's study, all patients were females (9). In Dawson's study, this method was performed for only 6 patients, among whom only one was female (10). In the current study, patients who met the inclusion criteria were within the age range of 19-57 years, with a mean of $35.25 \pm 9.78$ years. The mean age of the participants in various similar studies is different. In a study by Gorriz and colleagues with a sample size of 77 patients, participants were within the age range of 15-69 years with a mean of 45 years (11). In the study by Nassiri et al., the sample size included 12 patients, aged 14-54 years, with a mean age of 29.8 years (9). In the Cholongitas study, which included 165 patients, the mean of subjects' age was $48 \pm 15$ years (7). The comparison between these studies indicates that the age distribution varies, depending on the inclusion criteria. Based on TJLB findings in our study, one case was diagnosed with hemophilia and 23 patients presented with bleeding disorders and platelet count $<30,000$. Meanwhile in the study by Nassiri et al., all the patients had hemophilia (9). Also, in Dawson's study, conducted in a hemophilia treatment center, all the patients were diagnosed with congenital bleeding disorders (Von Willebrand's disease and hemophilia) and hepatitis C (and one case of HIV) (10). In addition, in Lee's study, in a sample size of 21 patients, all subjects had chronic hepatitis and cirrhosis, and 19 patients suffered from liver failure after bone marrow transplantation; in two other patients, the initial diagnosis was uncertain (12). In our study, performed on 24 patients, the sampling was successful in $87.5 \%$ of the cases, and adequate liver biopsy specimens were obtained via TJLB. Beckmann's study, conducted on 102 patients in 2009, indicated a technical success rate of $82 \%$ (13). However, the success rate in two studies by Miraglia et al. in 2006 (14) and Podolsky (7) was reported as $100 \%$. The success rate of a diagnostic intervention depends on several factors including the equipment, researcher's skills, indications, and inclusion criteria. Considering the reported results in other studies, the success rate in the present study was adequate. The intervention duration in our study was $23.80 \pm 9.34 \mathrm{~min}$, ranging from 10 to 40 minutes. The duration was consistent with that of other studies. However, in Gorriz's study, the average intervention duration was $48 \mathrm{~min}$ (11); also, in Miraglia's study, considering the extra time needed for frozen section, the average duration was $80 \mathrm{~min}(14)$. In our study, $37.5 \%$ of the patients had no complications and $62.5 \%$ had minor or major complications. The incidence rate of complications varies in different studies; only Miraglia's study, conducted on 17 patients, reported no adverse side-effects (14). Also, in Dawson's study, conducted on six patients, only one patient had complaints of pain in the right upper quadrant of the liver (10). Therefore, it can be concluded that occurrence of major complications is uncommon in this diagnostic method and most of the minor complications are self-limited and not threatening for the patient. Given that in the current study, neck puncture was performed under ultrasound guidance, no damage to the carotid or endothelium of internal jugular vein or neck hematoma was observed in our patients.

\section{Conclusions}

Ultrasound-guided TJLB can be an effective method if performed by an experienced radiologist. It is associated with a high success rate and almost no major complications. These biopsies, considering the small error percentage detected by pathologists, can be useful for patients who are contraindicated to other types of biopsy. Given that this study was performed on 24 patients, further research with greater sample size is recommended to accurately determine high-risk patients and other probable indications.

\section{Acknowledgments:}

This study is part of residency thesis of the first author and supported by Mashhad University of Medical Sciences. We would like to thank all our colleagues and Radiology department of Emam Reza hospital for collaboration and their commitment. 


\section{Conflict of Interest:}

There is no conflict of interest to be declared.

\section{Authors' contributions:}

All authors contributed to this project and article equally. All authors read and approved the final manuscript.

\section{References:}

1) Soyer P, Fargeaudou Y, Boudiaf M, Rymer R. Transjugular liver biopsy using ultrasonographic guidance for jugular vein puncture and an automated device for hepatic tissue sampling: a retrospective analysis of 200 consecutive cases. Abdom Imaging. 2008; 33(6): 627-32. doi: 10.1007/s00261-007-9357-3. PMID: 18180981.

2) Elahi B. Tranckal Anatomi. Jihon Publocation. 3ed. 2000. Tehran: 55-75, 198-224.

3) Miraglia R, Maruzzelli L, Minervini MI, Mamone G, Caruso S, Cintorino D, et al. Transjugular biopsy of an atypical focal nodular hyperplasia in a patient with acute liver failure clinical presentation. Emerg radiol. 2009; 16(3): 223-5. doi: 10.1007/s10140-008-0731-4. PMID: 18463903.

4) Parulekar SG, Bree LB. In: Liver. Goldberg Radiology. Philadelphia: Saunders. 2007; 339-410.

5) Habdank K, Restrepo R, Ng V, Connolly BL, Temple MJ, Amaral J, et al. Combined sonographic and fluoroscopic guidance during transjugular hepatic biopsies performed in children: A retrospective study of 74 biopsies. AJR Am J Roentgenol. 2003; 180(5): 1393-8. doi: 10.2214/ajr.180.5.1801393. PMID: 12704057.

6) Esposito AA, Nicolini A, Meregaglia D, Sangiovanni A, Biondetti P. Role of transjugular liver biopsy in the diagnostic and therapeutic management of patients with severe liver disease. Radiol Med. 2008; 113(7): 1008-17. doi: 10.1007/s11547-008-0311-4. PMID: 18781282.

7) Podolsky DK, Isselbacher KJ. Inflammatory Bowel Disease. In: Fauci A, Kasper D, Longo D, Braunwald E, hauser S, Hameson J editors. Harrison s principle of Internal Medicine. 17th ed. McGraw-Hill. New York. 2008: 562-625.

8) Mauro MA, Murphy KPJ, Thomson KR, Venbrux AC, Zollikofer CL. Image-Guided Interventions, 2nd Edition. Philadelpha: Saunders Elsevier. 2008: 233-56.

9) Nassiri TM, Ghanaati H, Azmoude A, Agdarkosh H, Safai SR, Lak M, et al. Assessment of safety and efficacy of transjugular liver biopsy as a diagnostic method in adult patients with congenital bleeding disorder with HCV infection. Iran J Radiol. 2004; 2(1-2): 73-9.

10) Dawson MA, McCarthy PH, Walsh ME, McLean CA, Thomson K, Roberts S, et al. Transjugular liver biopsy is a safe and effective intervention to guide management for patients with a congenital bleeding disorder infected with hepatitis C. Int Med J. 2005; 35(9): 556-9. doi: 10.1111/j.1445-5994.2005.00877.x. PMID: 16105158 .

11) Gorriz E, Reyes R, Lobrano MB, Pulido-Duque JM, San Roman JL, Lonjedo E, et al. Transjugular liver biopsy: a review of 77 biopsies using a spring-Propelled cutting needle (Biopsy Gun). Cardiovasc Int Radiol. 1996; 19(6): 442-5. PMID: 8994714.

12) Lee D, Chitturi S, Kench J, George J, Fuller S, Bradstock K, et al. Transjugular liver biopsy effecting changes in clinical management. Australas Radiol. 2003; 47(2): 117-20. PMID: 12780438.

13) Beckmann MG, Bahr MJ, Hadem J, Bredt M, Wedemeyer H, Schneider AS, et al. Clinical relevance of transjugular liver biopsy in comparison with percutaneous and laparoscopic liver biopsy. Gastroenterol Res Pract. 2009; 2009: 947014. doi: 10.1155/2009/947014. PMID: 19920868, PMCID: PMC2777243.

14) Miraglia R, Luca A, Gruttadauria S, Minervini MI, Vizzini G, Arcadipane A, et al. Contribution of transjugular liver biopsy in patients with the clinical presentation of acute liver failure. Cardiovasc Intervent Radiol. 2006; 29(6): 1008-10. doi: 10.1007/s00270-006-0052-5. PMID: 16967214. 